\title{
Surrogate endpoints and competing risk of death in cardiac arrest research
}

\author{
Victoria A. McCredie $e^{1,2,3^{*}}$ and Damon C. Scales ${ }^{1,2,3}$ \\ See related research by Donnino et al., http://ccforum.biomedcentral.com/articles/10.1186/s13054-016-1257-x
}

\begin{abstract}
We urgently need new therapies to improve outcomes after cardiac arrest. Initial studies typically target surrogate endpoints, and these studies help to inform subsequent larger trials that are powered to measure more patient-orientated clinical outcomes such as survival. The competing risk of death and premature assessment of neurological prognosis pose significant challenges to measuring these surrogate endpoints after cardiac arrest.
\end{abstract}

We urgently need new therapies to improve survival with good neurological outcomes after cardiac arrest [1]. Cardiac arrest is a major global health problem, with approximately 424,000 patients experiencing an out-ofhospital cardiac arrest each year in the USA [2]. Overall, survival rates appear to have increased marginally over time, but the majority of these patients will still die before hospital discharge [3]. Initial studies of new treatments typically target surrogate endpoints rather than survival; for example, reducing persistent precipitating pathology, organ dysfunction, and markers of secondary neurological injury [4]. Interventions that can be shown to modify rates of these surrogate endpoints can then be studied in larger, adequately powered trials targeting more meaningful clinical outcomes.

In this issue of Critical Care, Donnino and colleagues present the results of a randomized controlled trial targeting one such surrogate endpoint, the reversal of shock after cardiac arrest [1]. Specifically, they tested whether corticosteroids-in doses similar to those used in sepsis trials-could shorten duration of vasopressor

\footnotetext{
* Correspondence: victoria.mccredie@sunnybrook.ca

'Department of Critical Care Medicine, Sunnybrook Health Sciences Centre, 2075 Bayview Avenue, Room D108, Toronto, ON M4N 3M5, Canada ${ }^{2}$ Interdepartmental Division of Critical Care Medicine, University of Toronto, Toronto, Ontario, Canada

Full list of author information is available at the end of the article
}

administration [5]. The trial addresses an important clinical question, and the investigators conclude that hydrocortisone does not decrease time to shock reversal in post-cardiac arrest patients. Most readers will interpret these results as evidence that the biological signal-as measured by the surrogate endpoint of shock reversal-is insufficient to justify routine use of steroids in these patients.

However, some experts may offer other potential interpretations for the apparent lack of a biological effect, including timing of medication initiation or inclusion of patients without adrenal insufficiency. Another possible explanation is that many patients died before they could achieve shock reversal, rendering this surrogate endpoint unable to discriminate between responders and nonresponders. Indeed, more than two-thirds (34/50) of patients died before hospital discharge, and it is unclear how many died before shock reversal [1]. The researchers used appropriate analytical techniques to account for this competing risk, but even the most sophisticated analyses cannot detect a change in a surrogate endpoint when most patients die before they can experience it.

Using a surrogate endpoint becomes even more problematic when it does not actually sit on the causal pathway. Ideally, a surrogate endpoint should have a clear relationship as an intermediate event occurring between the exposure of interest and the more meaningful clinical outcome [6], in this case death. However, only one patient in each group died due to refractory shock. Similar to most cardiac arrest trials, the majority of deaths (68\%; 23/34) occurred after a decision to withdraw lifesustaining therapy—classified as a 'primary neurological withdrawal of care'. Decisions to limit life support treatments after cardiac arrest are usually based on predictions of neurological prognosis, and may have little to do with ongoing need for vasopressor support $[7,8]$. This further obfuscates the interpretation of shock reversal as a surrogate endpoint.

Current evidence-based guidelines now recommend delaying neurological prognostication for at least $72 \mathrm{~h}$ 
after return of spontaneous circulation owing to the inaccuracy of clinical examinations performed before this time point [9]. As a consequence, any clinical trial testing post-resuscitation interventions-including future trials testing effects on surrogate endpoints-will need to consider ways of ensuring that neurological prognostication is appropriately delayed and that decisions to withdraw life-sustaining therapy are not based solely on subjective determinations. This type of approach was successfully implemented in the Targeted Temperature Management trial, which serves as a model for future trials of post-arrest interventions [10]. To avoid the competing risk between surrogate endpoints and deaths related to estimates of poor neurological prognosis, future trials could consider restricting the time frame for measuring surrogate endpoints to earlier than $72 \mathrm{~h}$. Alternatively, trialists could choose surrogate endpoints that clearly lie on the causal pathway between the exposure of interest and subsequent decisions to withdraw life-sustaining therapy, which is the usual mode of death after cardiac arrest. These approaches would introduce additional design challenges, but could improve the interpretability of surrogate endpoints in future cardiac arrest trials.

\section{Funding}

DCS holds operating grants from the Canadian Institutes of Health Research.

\section{Authors' contributions}

VAM and DCS conceived the content of this editorial and drafted the manuscript. Both authors have read and approved the final manuscript.

\section{Competing interests}

The authors declare that they have no competing interests.

\section{Author details}

'Department of Critical Care Medicine, Sunnybrook Health Sciences Centre, 2075 Bayview Avenue, Room D108, Toronto, ON M4N 3M5, Canada. ${ }^{2}$ Interdepartmental Division of Critical Care Medicine, University of Toronto, Toronto, Ontario, Canada. ${ }^{3}$ Institute of Health Policy, Management and Evaluation, University of Toronto, Toronto, Ontario, Canada.

Published online: 29 June 2016

\section{References}

1. Donnino MW, Andersen LW, Berg KM, et al. Corticosteroid therapy in refractory shock following cardiac arrest: a randomized, double-blind, placebo-controlled, trial. Crit Care. 2016;20(1):82.

2. Go AS, Mozaffarian D, Roger VL, et al. Heart disease and stroke statistics - 2014 update: a report from the American Heart Association Circulation. 2014:129(3):e28-292.

3. Wong MK, Morrison L, Qiu F, et al. Trends in short- and long-term survival among out-of-hospital cardiac arrest patients alive at hospital arrival. Circulation. 2014;130(21):1883-90.

4. Nolan JP, Neumar RW, Adrie C, et al. Post-cardiac arrest syndrome: epidemiology, pathophysiology, treatment, and prognostication. A Scientific Statement from the International Liaison Committee on Resuscitation; the American Heart Association Emergency Cardiovascular Care Committee; the Council on Cardiovascular Surgery and Anesthesia; the Council on Cardiopulmonary, Perioperative, and Critical Care; the Council on Clinical Cardiology; the Council on Stroke. Resuscitation. 2008;79(3):350-79.

5. Annane D, Bellissant E, Bollaert PE, et al. Corticosteroids for treating sepsis. Cochrane Database Syst Rev. 2015;12:Cd002243.
6. Prentice RL. Surrogate endpoints in clinical trials: definition and operational criteria. Stat Med. 1989:8(4):431-40.

7. Elmer J, Torres C, Aufderheide TP, et al. Association of early withdrawal of life-sustaining therapy for perceived neurological prognosis with mortality after cardiac arrest. Resuscitation. 2016;102:127-35. doi: 10.1016/j. resuscitation.2016.01.016. Epub 2016 Feb 3.

8. Perman SM, Kirkpatrick JN, Reitsma AM, et al. Timing of neuroprognostication in postcardiac arrest therapeutic hypothermia. Crit Care Med. 2012:40(3):719-24.

9. Callaway CW, Donnino MW, Fink EL, et al. Part 8: post-cardiac arrest care: 2015 American Heart Association Guidelines update for cardiopulmonary resuscitation and emergency cardiovascular care. Circulation. 2015;132(18 Suppl 2):S465-82.

10. Nielsen N, Wetterslev J, Cronberg T, et al. Targeted temperature management at 33 degrees $\mathrm{C}$ versus 36 degrees $\mathrm{C}$ after cardiac arrest. $\mathrm{N}$ Engl J Med. 2013;369(23):2197-206. 